TRANSACTIONS OF THE

AMERICAN MATHEMATICAL SOCIETY

Volume 365, Number 6, June 2013, Pages 3309-3327

S 0002-9947(2013)05767-4

Article electronically published on January 4, 2013

\title{
THE NIELSEN REALIZATION PROBLEM FOR ASYMPTOTIC TEICHMÜLLER MODULAR GROUPS
}

\author{
EGE FUJIKAWA AND KATSUHIKO MATSUZAKI \\ Dedicated to Professor Masahiko Taniguchi on the occasion of his 60th birthday
}

\begin{abstract}
Under a certain geometric assumption on a hyperbolic Riemann surface, we prove an asymptotic version of the fixed point theorem for the Teichmüller modular group, which asserts that every finite subgroup of the asymptotic Teichmüller modular group has a common fixed point in the asymptotic Teichmüller space. For its proof, we use a topological characterization of the asymptotically trivial mapping class group, which has been obtained in the authors' previous paper, but a simpler argument is given here. As a consequence, every finite subgroup of the asymptotic Teichmüller modular group is realized as a group of quasiconformal automorphisms modulo coincidence near infinity. Furthermore, every finite subgroup of a certain geometric automorphism group of the asymptotic Teichmüller space is realized as an automorphism group of the Royden boundary of the Riemann surface. These results can be regarded as asymptotic versions of the Nielsen realization theorem.
\end{abstract}

\section{IntRoduCtion AND STATEMENT OF RESUlts}

We consider the group $\mathrm{QC}(R)$ of all quasiconformal automorphisms of a Riemann surface $R$, the quasiconformal mapping class group MCG $(R)$ of all homotopy equivalence classes of the elements of $\mathrm{QC}(R)$, and the surjective homomorphism $q: \mathrm{QC}(R) \rightarrow \operatorname{MCG}(R)$. The realization problem for the mapping class group is asking whether there exists a homomorphism $\mathcal{E}: \Gamma \rightarrow \mathrm{QC}(R)$ such that $q \circ \mathcal{E}=\left.\mathrm{id}\right|_{\Gamma}$ for a given subgroup $\Gamma$ of $\operatorname{MCG}(R)$. In particular, we refer to the realization problem for a finite subgroup of $\mathrm{MCG}(R)$ as the Nielsen realization problem.

For an analytically finite Riemann surface $R$ (in other words, for a hyperbolic surface $R$ of finite area), Kerckhoff [15] proved the fixed point theorem for a finite subgroup of the Teichmüller modular group $\operatorname{Mod}(R) \cong \operatorname{MCG}(R)$. It asserts that every finite subgroup of $\operatorname{Mod}(R)$ has a common fixed point in the Teichmüller space $T(R)$. This is equivalent to the statement that every finite subgroup of MCG $(R)$ can be realized as a group of conformal automorphisms of the Riemann surface corresponding to the common fixed point, and thus gives an affirmative answer to the Nielsen realization problem. The Nielsen realization problem is also true even for an analytically infinite Riemann surface. Indeed, a generalization of the fixed point theorem to analytically infinite Riemann surfaces follows from a result by Markovic 19.

In this paper, we consider an asymptotic version of the fixed point theorem for the asymptotic Teichmüller modular group. The asymptotic Teichmüller space $A T(R)$

Received by the editors May 22, 2011 and, in revised form, November 24, 2011.

2010 Mathematics Subject Classification. Primary 30F60, 32G15; Secondary 37F30, 30 F25.

Key words and phrases. Riemann surface, hyperbolic geometry, Teichmüller space, quasiconformal mapping class group.

(C)2013 American Mathematical Society 3309

Reverts to public domain 28 years from publication 
is defined in a similar manner to the Teichmüller space by replacing conformal equivalence with asymptotically conformal equivalence. Every element of MCG $(R)$ induces a biholomorphic automorphism of $A T(R)$, which gives a representation $\iota_{A T}$ from $\operatorname{MCG}(R)$ to the group $\operatorname{Aut}(A T(R))$ of all biholomorphic automorphisms of $A T(R)$. The asymptotic Teichmüller modular group $\operatorname{Mod}_{A T}(R)$ is defined to be the image of $\iota_{A T}$. Our fixed point theorem can be stated as follows.

Theorem 2.7. Every finite subgroup of $\operatorname{Mod}_{A T}(R)$ has a common fixed point in $A T(R)$ if $R$ satisfies the weak bounded geometry condition.

The weak bounded geometry condition is a condition of hyperbolic geometry on a Riemann surface $R$ by which we mean that there is a lower bound of injectivity radii on $R$ except for cusp neighborhoods and there is a connected subsurface in $R$ with an upper bound of injectivity radii that carries the fundamental group of $R$. We will prove Theorem 2.7 in Section 4 by using a topological characterization of the kernel of $\iota_{A T}$ as Theorem 3.2 in Section 3. We expect that the statement itself of Theorem 2.7 is valid without the assumption of the weak bounded geometry condition, but Theorem 3.2 definitely needs this assumption.

As a consequence of Theorem 2.7. we have two asymptotic versions of the Nielsen realization theorem. The first one is for the asymptotic Teichmüller modular group. The peripheral quasiconformal automorphism group $\mathrm{QC}_{e}(R)$ is the group of all peripheral equivalence classes of quasiconformal automorphisms of $R$. Here we say that two quasiconformal automorphisms of $R$ are peripherally equivalent if they are coincident outside some topologically finite subsurface of finite area in $R$. The projection is denoted by $e: \mathrm{QC}(R) \rightarrow \mathrm{QC}_{e}(R)$, which induces a surjective homomorphism $q_{e}: \mathrm{QC}_{e}(R) \rightarrow \operatorname{Mod}_{A T}(R)$. Then the realization problem for the asymptotic Teichmüller modular group is asking whether there exists a local section of $q_{e}$ for a given subgroup of $\operatorname{Mod}_{A T}(R)$. The following theorem says that this problem is true for every finite subgroup of $\operatorname{Mod}_{A T}(R)$ under the same geometric assumption on $R$, and thus this can be regarded as an asymptotic version of the Nielsen realization theorem.

Theorem 5.2. Let $R$ be a Riemann surface satisfying the weak bounded geometry condition. For every finite subgroup $\hat{\Gamma}$ of $\operatorname{Mod}_{A T}(R)$, there exists a homomorphism $\mathcal{E}_{e}: \hat{\Gamma} \rightarrow \mathrm{QC}_{e}(R)$ such that $q_{e} \circ \mathcal{E}_{e}=\left.\mathrm{id}\right|_{\hat{\Gamma}}$.

In fact, every finite subgroup $\hat{\Gamma}$ of $\operatorname{Mod}_{A T}(R)$ can be realized as a subgroup of $\mathrm{QC}_{e}(R)$ whose elements are conformal outside some topologically finite subsurface of finite area in the Riemann surface corresponding to the common fixed point. We demonstrate these arguments in Section 5.

Another asymptotic version of the Nielsen realization theorem is for the boundary geometric automorphism group of the asymptotic Teichmüller space. We consider the Royden boundary $d R$ of $R$, which is the ideal boundary of the minimal compactification of $R$ to which a certain family of functions on $R$ extend continuously. We use a subset $d_{0} R$ of $d R$ obtained by removing all boundary points corresponding to punctures of $R$. Every quasiconformal automorphism of $R$ extends to a homeomorphism of $d R$ and keeps $d_{0} R$ invariant.

Let Homeo* $\left(d_{0} R\right)$ denote the group of all homeomorphic automorphisms of $d_{0} R$ that extend continuously to quasiconformal homeomorphisms of some neighborhoods of $d_{0} R$. This quasiconformal homeomorphism on the neighborhood of $d_{0} R$ is 
called a supporting map for the corresponding element of Homeo* $\left(d_{0} R\right)$. The supporting map is uniquely determined up to the difference outside some neighborhood of $d_{0} R$, which defines an injective homomorphism $\nu: \mathrm{QC}_{e}(R) \rightarrow \operatorname{Homeo}^{*}\left(d_{0} R\right)$. A supporting map also induces a biholomorphic automorphism of $A T(R)$. We define the boundary geometric automorphism group $\operatorname{Aut}^{*}(A T(R))$ as the group of all biholomorphic automorphisms of $A T(R)$ induced by supporting maps. Then we have a surjective homomorphism $q_{b}$ from Homeo* $\left(d_{0} R\right)$ to $\operatorname{Aut}^{*}(A T(R))$. Here is a commutative diagram for the homomorphisms we have obtained, where $i$ stands for the inclusion map:

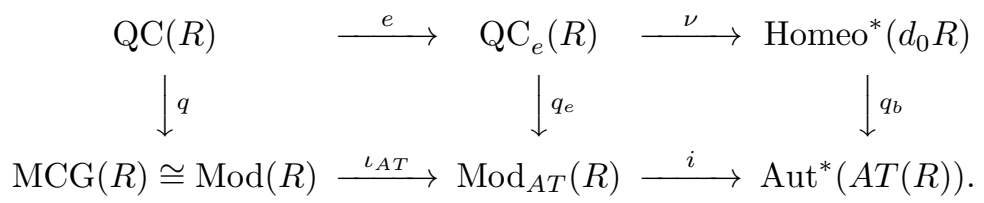

The realization problem for the boundary geometric automorphism group is asking whether there exists a local section of $q_{b}$ for a given subgroup of $\operatorname{Aut}^{*}(A T(R))$. The following theorem states that this problem is true for every finite subgroup of Aut $^{*}(A T(R))$ under the same geometric assumption on $R$ as before.

Theorem 6.5. Let $R$ be a Riemann surface satisfying the weak bounded geometry condition. For every finite subgroup $\widetilde{\Gamma}$ of $\operatorname{Aut}^{*}(A T(R))$, there exists a homomorphism $\mathcal{E}_{b}: \widetilde{\Gamma} \rightarrow$ Homeo $^{*}\left(d_{0} R\right)$ such that $q_{b} \circ \mathcal{E}_{b}=\left.\mathrm{id}\right|_{\widetilde{\Gamma}}$.

We will see this version of the realization theorem using the Royden boundary in Section 6. Finally, in Section 7, we introduce the space of ends of $R$ and deal with the pure mapping class group, which is defined to be a subgroup of $\operatorname{MCG}(R)$ consisting of all elements fixing each non-cuspidal end.

\section{Statement of the Fixed Point theorem}

In this section, we first review the fixed point theorem for the Teichmüller modular group and the Nielsen realization theorem for the quasiconformal mapping class group, especially for analytically infinite Riemann surfaces. Then we explain our fixed point theorem for the asymptotic Teichmüller modular group.

Throughout this paper, we assume that a Riemann surface $R$ admits a hyperbolic structure, that is, $R$ is represented as the quotient space $\mathbb{H} / H$ of the hyperbolic plane $\mathbb{H}$ by a torsion-free Fuchsian group $H$. Let $\mathrm{QC}(R)$ be the group of all quasiconformal automorphisms of $R$. A quasiconformal mapping class of $R$ is the homotopy equivalence class $[g]$ of quasiconformal automorphisms $g \in \mathrm{QC}(R)$, and the quasiconformal mapping class group $\mathrm{MCG}(R)$ of $R$ is the group of all quasiconformal mapping classes of $R$. Here the homotopy is considered to be relative to the ideal boundary at infinity $\partial R=(\partial \mathbb{H}-\Lambda(H)) / H$, where $\Lambda(H)$ is the limit set of $H$. The correspondence $g \mapsto[g]$ gives a surjective homomorphism $q: \mathrm{QC}(R) \rightarrow \operatorname{MCG}(R)$. The realization problem for the quasiconformal mapping class group $\operatorname{MCG}(R)$ is asking whether there exists a homomorphism $\mathcal{E}: \Gamma \rightarrow \mathrm{QC}(R)$ such that $q \circ \mathcal{E}=\left.\mathrm{id}\right|_{\Gamma}$ for a given subgroup $\Gamma$ of $\mathrm{MCG}(R)$.

The quasiconformal mapping class group acts on the Teichmüller space. The Teichmüller space $T(R)$ of a Riemann surface $R$ is the set of all equivalence classes $[f]$ of quasiconformal homeomorphisms $f$ of $R$. Here we say that two quasiconformal homeomorphisms $f_{1}$ and $f_{2}$ of $R$ are equivalent if there exists a conformal 
homeomorphism $h: f_{1}(R) \rightarrow f_{2}(R)$ such that $f_{2}^{-1} \circ h \circ f_{1}$ is homotopic to the identity on $R$. Here the homotopy is again considered to be relative to the ideal boundary at infinity $\partial R$. The Teichmüller space $T(R)$ can be embedded in the complex Banach space of all bounded holomorphic quadratic differentials on $R^{-}$, where $R^{-}$is the complex conjugate of $R$. In this way, $T(R)$ is endowed with the complex structure. For details, see [16] and [26].

Every element $[g] \in \operatorname{MCG}(R)$ induces a biholomorphic automorphism $[g]_{*}$ of $T(R)$ by $[f] \mapsto\left[f \circ g^{-1}\right]$, which is also isometric with respect to the TeichmüllerKobayashi distance. Let $\operatorname{Aut}(T(R))$ denote the group of all biholomorphic automorphisms of $T(R)$. Then we have a homomorphism

$$
\iota_{T}: \operatorname{MCG}(R) \rightarrow \operatorname{Aut}(T(R))
$$

given by $[g] \mapsto[g]_{*}$, and we define the Teichmüller modular group by

$$
\operatorname{Mod}(R)=\iota_{T}(\operatorname{MCG}(R)) .
$$

We call an element of $\operatorname{Mod}(R)$ a Teichmüller modular transformation. It is proved in Earle, Gardiner and Lakic 22 that the homomorphism $\iota_{T}$ is injective (faithful) for all Riemann surfaces $R$ of non-exceptional type. See also [6] and [22] for other proofs. Here we say that a Riemann surface $R$ is of exceptional type if $R$ has finite hyperbolic area and satisfies $2 g+n \leq 4$, where $g$ is the genus of $R$ and $n$ is the number of punctures of $R$. It was a problem to determine if the homomorphism $\iota_{T}$ is also surjective, especially for an analytically infinite Riemann surface. In Markovic [18, the final step of this problem has been solved affirmatively, and thus we have the following.

Theorem 2.1. For a Riemann surface $R$ of non-exceptional type, $\operatorname{MCG}(R) \cong$ $\operatorname{Mod}(R)=\operatorname{Aut}(T(R))$.

The Nielsen realization problem is the realization problem for a finite subgroup of $\operatorname{MCG}(R)$; namely, it is asking whether there exists a homomorphism $\mathcal{E}: \Gamma \rightarrow$ $\mathrm{QC}(R)$ such that $q \circ \mathcal{E}=\left.\mathrm{id}\right|_{\Gamma}$ for a finite subgroup $\Gamma$ of $\operatorname{MCG}(R)$. For a compact Riemann surface $R$, Nielsen himself proved that a finite cyclic subgroup $\Gamma$ can always be realized, and after several partial solutions by Fenchel and Zieschang, Kerckhoff [15] finally proved the following fixed point theorem, which gives an affirmative answer to the Nielsen realization problem.

Theorem 2.2. Let $R$ be an analytically finite Riemann surface. Then a subgroup of $\operatorname{Mod}(R)$ is finite if and only if it has a common fixed point in $T(R)$.

Since a Teichmüller modular transformation having a fixed point $p=[f] \in$ $T(R)$ is realized as a conformal automorphism of the Riemann surface $R_{p}=f(R)$ corresponding to $p$, Theorem 2.2 is equivalent to the statement that, if a subgroup of $\operatorname{MCG}(R)$ is finite, then it can be realized as a group of conformal automorphisms of $R_{p}$ (and vice versa). Since the groups $\mathrm{QC}(R)$ and $\mathrm{QC}\left(R_{p}\right)$ are quasiconformally conjugate, this implies that there exists a homomorphism $\mathcal{E}: \Gamma \rightarrow \mathrm{QC}(R)$ such that $q \circ \mathcal{E}=\left.\mathrm{id}\right|_{\Gamma}$ for every finite group $\Gamma$ of $\operatorname{MCG}(R)$.

A generalization of Theorem 2.2 to analytically infinite Riemann surfaces follows from the result on uniformly quasisymmetric groups by Markovic [19].

Theorem 2.3. Let $R$ be a Riemann surface in general. For a subgroup $G$ of $\operatorname{Mod}(R)$, the orbit $G(p)$ is bounded for some $p \in T(R)$ if and only if $G$ has a 
common fixed point in $T(R)$. In particular, a finite subgroup of $\operatorname{Mod}(R)$ has a common fixed point in $T(R)$.

Hence Theorem 2.3 implies the following statement, and eventually the Nielsen realization problem is true for all Riemann surfaces.

Corollary 2.4. Let $R$ be a Riemann surface in general. Every finite subgroup $\Gamma$ of $\operatorname{MCG}(R)$ can be realized as a group of conformal automorphisms of some Riemann surface that is quasiconformally equivalent to $R$. In particular, there exists a homomorphism $\mathcal{E}: \Gamma \rightarrow \mathrm{QC}(R)$ such that $q \circ \mathcal{E}=\left.\mathrm{id}\right|_{\Gamma}$ for every finite subgroup $\Gamma$ of $\mathrm{MCG}(R)$.

Note that the entire quasiconformal mapping class group $\Gamma=\operatorname{MCG}(R)$ cannot be lifted into the group $\mathrm{QC}(R)$ of the quasiconformal homeomorphisms of $R$. Indeed, for a compact Riemann surface $R$ of genus $g \geq 2$, there exists no homomorphism $\mathcal{E}: \operatorname{MCG}(R) \rightarrow \mathrm{QC}(R)$ such that $q \circ \mathcal{E}=\mathrm{id}$. See [20] and [21].

In what follows, we consider an asymptotic version of the fixed point theorem for the asymptotic Teichmüller modular group. The asymptotic Teichmüller space has been introduced in Gardiner and Sullivan 14. for the unit disk and in Earle, Gardiner and Lakic [3] and [4 for an arbitrary Riemann surface. We say that a quasiconformal homeomorphism $f$ of $R$ is asymptotically conformal if, for every $\epsilon>0$, there exists a compact subset $V$ of $R$ such that the maximal dilatation $K\left(\left.f\right|_{R-V}\right)$ of the restriction of $f$ to $R-V$ is less than $1+\epsilon$. We say that two quasiconformal homeomorphisms $f_{1}$ and $f_{2}$ of $R$ are asymptotically equivalent if there exists an asymptotically conformal homeomorphism $h: f_{1}(R) \rightarrow f_{2}(R)$ such that $f_{2}^{-1} \circ h \circ f_{1}$ is homotopic to the identity on $R$ relative to the ideal boundary at infinity $\partial R$. The asymptotic Teichmüller space $A T(R)$ of $R$ is the set of all asymptotic equivalence classes [[f]] of quasiconformal homeomorphisms $f$ of $R$.

The asymptotic Teichmüller space $A T(R)$ is of interest only when $R$ is analytically infinite. Otherwise $A T(R)$ is trivial, that is, it consists of just one point. Since a conformal homeomorphism is asymptotically conformal, there is a projection $\alpha: T(R) \rightarrow A T(R)$ that maps each Teichmüller equivalence class $[f] \in T(R)$ to the asymptotic Teichmüller equivalence class $[[f]] \in A T(R)$. The asymptotic Teichmüller space $A T(R)$ has a complex structure such that $\alpha$ is holomorphic. See also [5] and [13].

Every element $[g] \in \operatorname{MCG}(R)$ induces a biholomorphic automorphism $[g]_{* *}$ of $A T(R)$ by $[[f]] \mapsto\left[\left[f \circ g^{-1}\right]\right]$, which is also isometric with respect to the asymptotic Teichmüller distance. See [4. Let $\operatorname{Aut}(A T(R))$ be the group of all biholomorphic automorphisms of $A T(R)$. Then we have a homomorphism

$$
\iota_{A T}: \operatorname{MCG}(R) \rightarrow \operatorname{Aut}(A T(R))
$$

given by $[g] \mapsto[g]_{* *}$, and we define the asymptotic Teichmüller modular group (the geometric automorphism group of $A T(R))$ by

$$
\operatorname{Mod}_{A T}(R)=\iota_{A T}(\operatorname{MCG}(R)) .
$$

We call an element of $\operatorname{Mod}_{A T}(R)$ an asymptotic Teichmüller modular transformation. It is different from the case of the representation $\iota_{T}: \operatorname{MCG}(R) \rightarrow \operatorname{Aut}(T(R))$ that the homomorphism $\iota_{A T}$ is not injective; namely, $\operatorname{Ker} \iota_{A T} \neq\{[\mathrm{id}]\}$ unless $R$ is either the unit disk or the once-punctured disk ([2]).

Definition 2.5. We call an element of Ker $\iota_{A T}$ asymptotically trivial and call Ker $\iota_{A T}$ the asymptotically trivial mapping class group. 
For example, if $R$ has a sequence of simple closed geodesics whose lengths tend to zero, a quasiconformal mapping class caused by the Dehn twists along all these curves is asymptotically trivial. On the other hand, any conformal mapping class of infinite order is not asymptotically trivial ([25]). We will give a topological characterization of an asymptotically trivial mapping class in the next section.

To formulate our results, we need to introduce the following geometric conditions on the hyperbolic structure of an analytically infinite Riemann surface. Hereafter, $\dot{R}$ denotes the non-cuspidal part of $R$ obtained by removing all horocyclic cusp neighborhoods of area one. Also, for a constant $M>0, R_{M}$ denotes the set of all points $x$ in $R$ satisfying a property that there exists a homotopically non-trivial and non-cuspidal closed curve based at $x$ whose hyperbolic length is less than $M$.

Definition 2.6. We say that a hyperbolic Riemann surface $R$ satisfies the bounded geometry condition if the following three conditions are fulfilled:

(i) lower bound condition: there exists a constant $m>0$ such that, for every point $x \in \dot{R}$, every homotopically non-trivial curve based at $x$ has hyperbolic length greater than or equal to $m$;

(ii) upper bound condition: there exist a constant $M>0$ and a connected component $R_{M}^{0}$ of $R_{M}$ such that the inclusion map $R_{M}^{0} \hookrightarrow R$ induces a surjective homomorphism $\pi_{1}\left(R_{M}^{0}\right) \rightarrow \pi_{1}(R)$;

(iii) $R$ has no ideal boundary at infinity $\partial R$.

Moreover, we say that $R$ satisfies the weak bounded geometry condition if only two conditions (i) and (ii) above are satisfied; namely, $R$ may have an ideal boundary at infinity.

Every normal cover that is not the universal cover of an analytically finite Riemann surface satisfies the bounded geometry condition and every normal cover that is not the universal cover of a topologically finite Riemann surface satisfies the weak bounded geometry condition. Moreover, if the convex core of a Riemann surface $R$ admits such pants decomposition that the diameter of the non-cuspidal part of each pair of pants (possibly degenerate) is uniformly bounded, then $R$ satisfies the weak bounded geometry condition. The bounded geometry condition is preserved under quasiconformal homeomorphisms. Thus, this can be regarded as a condition for the Teichmüller space.

In [11, we have proved that every element of $\operatorname{Mod}_{A T}(R)$ of finite order has a fixed point in $A T(R)$ if $R$ satisfies a certain stronger bounded geometry condition. Our main theorem in this paper is an extension of this result to the following asymptotic version of the fixed point theorem.

Theorem 2.7 (Fixed point theorem). Let $R$ be a Riemann surface satisfying the weak bounded geometry condition. Then every finite subgroup of $\operatorname{Mod}_{A T}(R)$ has a common fixed point in $A T(R)$.

We prove Theorem 2.7 in Section 4 after some preparation in Section 3.

\section{TOPOLOGICAL CHARACTERIZATION OF THE ASYMPTOTICALLY TRIVIAL MAPPING CLASS GROUP}

For the proof of Theorem 2.7 a topological characterization of the asymptotically trivial mapping class group plays a central role. To state this result and give a proof for it, we begin with several definitions. 
By a subsurface of a Riemann surface $R$, we mean a surface possibly with boundary in $R$. We say that a subsurface $V$ of $R$ is topologically finite if its fundamental group is finitely generated and, in addition, if each component of the relative boundary $\partial V \subset R$ is homeomorphic to a circle.

For a homotopically non-trivial and non-cuspidal simple closed curve $c$ on a hyperbolic Riemann surface $R$, let $c_{*}$ denote the unique simple closed geodesic that is freely homotopic to $c$. For a subsurface $V$ of $R$ whose relative boundary $\partial V$ consists of simple closed curves, let $V_{*}$ denote a subsurface of $R$ each of whose relative boundary components is the simple closed geodesic that is freely homotopic to the corresponding component of $\partial V$. Note that, if a relative boundary component of $\partial V$ is homotopically trivial or cuspidal, then we assume that the corresponding component of $\partial V_{*}$ degenerates.

We consider the following subgroup of the quasiconformal mapping class group.

Definition 3.1. The stable quasiconformal mapping class group $G_{\infty}(R)$ is a subgroup of $\operatorname{MCG}(R)$ consisting of all essentially trivial mapping classes $[g]$ of a Riemann surface $R$. Here $[g] \in \operatorname{MCG}(R)$ is said to be essentially trivial (or trivial near infinity) if there exists a topologically finite subsurface $V$ of finite area in $R$ such that, for each connected component $U$ of $R-V$, the restriction $\left.g\right|_{U}: U \rightarrow R$ is homotopic to the inclusion map id $\left.\right|_{U}: U \hookrightarrow R$ relative to the ideal boundary at infinity.

For example, any quasiconformal mapping class given by the composition of finitely many Dehn twists is essentially trivial. The stable quasiconformal mapping class group consists of countably many elements.

It is obvious from the definition that $G_{\infty}(R) \subset \operatorname{Ker} \iota_{A T}$. This inclusion is not necessarily an equality, in general. See [9, Remark 4.1] for an example where this inclusion is not an equality. However, when $R$ satisfies the weak bounded geometry condition, we have the equality, which gives the topological characterization of $\operatorname{Ker} \iota_{A T}$.

Theorem 3.2. Let $R$ be a Riemann surface satisfying the weak bounded geometry condition. Then $\operatorname{Ker} \iota_{A T}=G_{\infty}(R)$.

In [11, this theorem was proved in an indirect way. In this section, we give a rather direct proof for it by summarizing necessary results proved in [11. Note that, in the previous paper, we used the upper bound condition in a stronger sense in that every point of $R$ has uniformly bounded injectivity radius (and hence there is no ideal boundary at infinity) and imposed this condition on $R$ together with the lower bound condition. This is because we avoided inessential complexity in the entire argument. However, only to prove Theorem 3.2, we can replace the stronger upper bound condition with the upper bound condition introduced in Section 2 . Actually, it is easy to verify that Lemma 4.9 in [11, which is used in the proof of Proposition 3.3 below, is still valid under this upper bound condition.

A $\Theta$-graph $X=(\vec{c}, \eta)$ in a hyperbolic Riemann surface $R$ is defined by an oriented simple closed geodesic $\vec{c}$ together with a non-degenerate oriented simple geodesic arc $\eta$ connecting $\vec{c}$ to itself perpendicularly and having no intersection with $\vec{c}$ except for its end points. (In [11, we have used a similar object to a $\Theta$-graph but a more general one called thetaframe.) Furthermore, we say that $X=(\vec{c}, \eta)$ is a $D$-bounded $\Theta$-graph for a constant $D>0$ if the hyperbolic lengths of $\vec{c}$ and $\eta$ are not greater than $D$. For a quasiconformal homeomorphism $f$ of $R$ onto another 
Riemann surface $R^{\prime}$ and for a $\Theta$-graph $X=(\vec{c}, \eta)$ in $R$, we denote by $f(X)_{*}$ the $\Theta$ graph in $R^{\prime}$ that is homotopic to $f(X)=(f(\vec{c}), f(\eta))$. More precisely, the $\Theta$-graph $f(X)_{*}=\left(f(\vec{c})_{*}, f(\eta)_{*}\right)$ consists of the oriented simple closed geodesic $f(\vec{c})_{*}$ freely homotopic to $f(\vec{c})$ and the oriented geodesic arc $f(\eta)_{*}$ defined as follows. Let $f\left(x_{1}\right)$ and $f\left(x_{2}\right)$ be the end points of $f(\eta)$ in $f(\vec{c})$ and let $H_{t}(0 \leq t \leq 1)$ be a homotopy sending $f(\vec{c})$ to $f(\vec{c})_{*}$. Then $H_{t}\left(f\left(x_{i}\right)\right)$ defines an arc $s_{i}$ from $f\left(x_{i}\right)$ to a point in $f(\vec{c})_{*}$ for $i=1,2$. The geodesic arc $f(\eta)_{*}$ connects $f(\vec{c})_{*}$ to itself in the homotopy class of $s_{1}^{-1} \cdot f(\eta) \cdot s_{2}$ with the end points throughout on $f(\vec{c})_{*}$.

The next proposition, which has been essentially proved in [11, ensures that we can always take a $\Theta$-graph that is not fixed by a non-trivial quasiconformal mapping class.

Proposition 3.3. Let $R$ be a Riemann surface satisfying the weak bounded geometry condition, and $U_{*}$ a topologically infinite subsurface of $R$ with geodesic boundary. Let $g$ be a quasiconformal automorphism of $R$ such that the restriction $\left.g\right|_{U_{*}}$ is not homotopic to the inclusion map $U_{*} \hookrightarrow R$, where the homotopy is not necessarily relative to the ideal boundary at infinity. Then there exists a $D$-bounded $\Theta$-graph $X$ in the $B$-neighborhood of $U_{*}$ such that $g(X)_{*} \neq X$. Here $D$ and $B$ are constants depending only on the constants $m$ and $M$ for the lower and upper bound conditions.

Proof. Let $R_{M}^{0}$ be the connected component of $R_{M}$ in the definition of the upper bound condition. We choose a point $x$ in $U_{*} \cap R_{M}^{0}$ sufficiently far away from the relative boundary $\partial U_{*}$ and a non-trivial, non-cuspidal simple loop $c_{x}$ based at $x$ whose length is less than $M$. Clearly $c_{x}$ is contained in $R_{M}^{0}$. Let $c$ be the simple closed geodesic freely homotopic to $c_{x}$. Then the length of $c$ is between $m$ and $M$, and $c$ is also contained in $R_{M}^{0}$. Moreover, there is a constant $B>0$ depending only on $m$ and $M$ such that the distance between $c_{x}$ and $c$ is not greater than $B$. Since $x$ is sufficiently far away from $\partial U_{*}$, we may assume that $c$ is contained in $U_{*}$. By [8. Proposition 3.1], giving an orientation to $c$ and choosing a suitable geodesic arc $\eta$, we have a $D$-bounded $\Theta$-graph $X=(\vec{c}, \eta)$ for a constant $D>0$ depending only on $m$ and $M$. We may also assume that $X$ is contained in $U_{*}$.

If $g(X)_{*} \neq X$, then the $\Theta$-graph $X$ is a desired one. If $g(X)_{*}=X$, then applying [11, Lemma 4.9] and modifying the obtained frame to a $\Theta$-graph (see [11, Proposition 4.3]), we can find a $\widetilde{D}$-bounded $\Theta$-graph $\widetilde{X}$ in the $\widetilde{B}$-neighborhood of $U_{*}$ such that $g(\widetilde{X})_{*} \neq \widetilde{X}$. Here the constants $\widetilde{D}$ and $\widetilde{B}$ again depend only on $m$ and $M$.

The following proposition is crucial for our proof of Theorem 3.2 This has been proved in [11, Lemma 7.3]. Roughly speaking, if there is an infinite family of mutually disjoint simple closed geodesics $\left\{c_{n}\right\}$ of moderate lengths that is mapped to a completely different family by a mapping class $[g]$, then, for a hyperbolic structure on $R$ in which $\left\{c_{n}\right\}$ are exactly the family of all short simple closed geodesics, $[g]$ cannot be realized as an asymptotically conformal automorphism.

Proposition 3.4. Let $R$ be a Riemann surface satisfying the lower bound condition, and $\left\{c_{n}\right\}_{n=1}^{\infty}$ a sequence of mutually disjoint simple closed geodesics on $R$ such that the hyperbolic lengths of $c_{n}$ are uniformly bounded from above. Let $[g] \in \operatorname{MCG}(R)$ be a quasiconformal mapping class such that $g\left(c_{n}\right)_{*} \neq c_{n^{\prime}}$ for any $n$ and $n^{\prime}$. Then $[g] \notin \operatorname{Ker} \iota_{A T}$. 
Now we are ready to give the proof for our theorem.

Proof of Theorem 3.2. Let $R$ be a Riemann surface satisfying the weak bounded geometry condition for the constants $m$ and $M$. We may assume that $R$ is topologically infinite. We take a quasiconformal mapping class $[g]$ that does not belong to $G_{\infty}(R)$, and prove that $[g] \notin \operatorname{Ker} \iota_{A T}$. The assumption $[g] \notin G_{\infty}(R)$ implies that, for each topologically finite subsurface $V$ of finite area in $R$, there exists a connected component $U$ of $R-V$ such that the restriction $\left.g\right|_{U_{*}}$ to the subsurface $U_{*}$ with geodesic boundary is not homotopic to the inclusion map id $\left.\right|_{U_{*}}: U_{*} \hookrightarrow R$ relative to the ideal boundary at infinity.

First, we consider the case where $R$ has no ideal boundary at infinity. Then by Proposition 3.3, there exists a $D$-bounded $\Theta$-graph $X$ in the $B$-neighborhood of $U_{*}$ such that $g(X)_{*} \neq X$. Let $\left\{R_{n}\right\}_{n=1}^{\infty}$ be a regular exhaustion of $R$. Namely, $\left\{R_{n}\right\}_{n=1}^{\infty}$ is an increasing sequence of compact subsurfaces $R_{n}$ such that $R=\bigcup_{n=1}^{\infty} R_{n}$ and each connected component of the complement $R-R_{n}$ is not relatively compact. Then, for each $n \geq 1$, there exists a $D$-bounded $\Theta$-graph $X_{n}=\left(\vec{c}_{n}, \eta_{n}\right)$ in the $B$-neighborhood of $\left(U_{n}\right)_{*}$ for some topologically infinite connected component $U_{n}$ of $R-R_{n}$ such that $g\left(X_{n}\right)_{*} \neq X_{n}$.

We will choose a sequence of simple closed geodesics $\left\{\tilde{c}_{n}\right\}_{n=1}^{\infty}$ of uniformly bounded lengths tending to the infinity of $R$ (that is, escaping from each compact subsurface of $R$ ) and satisfying $g\left(\tilde{c}_{n}\right)_{*} \neq \tilde{c}_{n}$ as follows. Fix $n$. For the $\Theta$-graph $X_{n}=\left(\vec{c}_{n}, \eta_{n}\right)$, if the non-oriented simple closed curve $c_{n}$ underlying $\vec{c}_{n}$ satisfies $g\left(c_{n}\right)_{*} \neq c_{n}$, then we just set $\tilde{c}_{n}=c_{n}$. Hence, hereafter, we only consider the case where $g\left(c_{n}\right)_{*}=c_{n}$. The assumption $g\left(X_{n}\right)_{*} \neq X_{n}$ implies $g\left(\eta_{n}\right)_{*} \neq \eta_{n}$ in this case. The two end points of $\eta_{n}$ on $c_{n}$ divide $c_{n}$ into two subarcs $\alpha_{n}^{1}$ and $\alpha_{n}^{2}$. Let $c_{n}^{i}$ be a closed curve $\alpha_{n}^{i} \cdot \eta_{n}$ for each $i=1,2$, which has the length bounded by $2 D$. If one of $\left\{c_{n}^{i}\right\}_{i=1,2}$, say $c_{n}^{1}$, is freely homotopic to a simple closed geodesic $\left(c_{n}^{1}\right)_{*}$, then we have $g\left(c_{n}^{1}\right)_{*} \neq\left(c_{n}^{1}\right)_{*}$ by $g\left(\eta_{n}\right)_{*} \neq \eta_{n}$. We set $\tilde{c}_{n}=\left(c_{n}^{1}\right)_{*}$ in this case.

Now we only deal with the case where each of $\left\{c_{n}^{i}\right\}_{i=1,2}$ is cuspidal, that is, freely homotopic to a simple closed curve around a puncture. Then a connected component of $R-c_{n}$ must be a twice punctured disk $P_{n}$. Consider a half-collar $A_{n}$ of $c_{n}$ in $R-P_{n}$, which is a ring domain within some distance $\omega$ from $c_{n}$. By the bounded geometry condition for $R$, the maximal width $\omega$ for such $A_{n}$ is uniformly bounded by a constant $D^{\prime}>0$ depending only on $m$ and $M$. See [8. Proposition 3.1] for this argument. This implies that the length of the shortest geodesic arc $\eta_{n}^{\prime}$ connecting $c_{n}$ to itself in $R-P_{n}$ is bounded by $2 D^{\prime}$ from above. Then we take the other $\Theta$-graph $X_{n}^{\prime}=\left(\vec{c}_{n}, \eta_{n}^{\prime}\right)$ and form the simple closed curves ${c^{\prime}}_{n}^{i}=\alpha_{n}^{i} \cdot \eta_{n}^{\prime}$ in the same way. Note that, in this case, at least one of $\left\{c_{n}^{i}\right\}_{i=1,2}$ must be non-cuspidal, for otherwise, $R$ would be a four-times punctured sphere.

If $g\left(X_{n}^{\prime}\right)_{*}=X_{n}^{\prime}$, then we choose a simple closed curve of length not greater than $2\left(D+D^{\prime}\right)$, which is composed of $\eta_{n}$ and $\eta_{n}^{\prime}$ together with the subarcs in $c_{n}$ connecting the end points of $\eta_{n}$ and $\eta_{n}^{\prime}$ suitably. We set the geodesic realization of this simple closed curve by $\tilde{c}_{n}$. Then $g\left(\tilde{c}_{n}\right)_{*} \neq \tilde{c}_{n}$. If $g\left(X_{n}^{\prime}\right)_{*} \neq X_{n}^{\prime}$, then at least one of $\left\{c_{n}^{\prime}\right\}_{i=1,2}$ has the geodesic realization $\left(c_{n}^{\prime}{ }_{n}^{i}\right)_{*}$, which is defined to be $\tilde{c}_{n}$. Since $g\left(\eta_{n}^{\prime}\right)_{*} \neq \eta_{n}^{\prime}$, we have $g\left(\tilde{c}_{n}\right)_{*} \neq \tilde{c}_{n}$. Thus we have checked all possibilities.

By passing to a subsequence, we may assume that the sequence $\left\{\tilde{c}_{n}\right\}_{n=1}^{\infty}$ of simple closed geodesics are mutually disjoint as well as each $g\left(\tilde{c}_{n}\right)_{*}$ is disjoint from $\tilde{c}_{n^{\prime}}$ for every $n^{\prime} \neq n$. Also by $g\left(\tilde{c}_{n}\right)_{*} \neq \tilde{c}_{n}$, we see that $g\left(\tilde{c}_{n}\right)_{*} \neq \tilde{c}_{n^{\prime}}$ for any $n$ and $n^{\prime}$. Then we apply Proposition 3.4 to conclude that $[g] \notin \operatorname{Ker} \iota_{A T}$. 
Next, we consider the case where $R$ has ideal boundary at infinity $\partial R$. If there exists a $D$-bounded $\Theta$-graph $X$ in the $B$-neighborhood of $U_{*}$ such that $g(X)_{*} \neq X$, then the proof can be carried out in the same way as above. If there exists no such $\Theta$-graph, then Proposition 3.3 implies that $\left.g\right|_{U_{*}}$ is homotopic to the inclusion map $\left.\mathrm{id}\right|_{U_{*}}: U_{*} \hookrightarrow R$. However, since $\left.g\right|_{U_{*}}$ is not homotopic to the inclusion map relative to the ideal boundary at infinity, $g$ is not the identity on $\partial R$. Then we apply the following lemma to complete the proof.

Lemma 3.5. Let $R$ be a Riemann surface with ideal boundary at infinity $\partial R$. Then a mapping class $[g] \in \mathrm{MCG}(R)$ induced by a quasiconformal automorphism $g \in \mathrm{QC}(R)$ that is not the identity on $\partial R$ is not asymptotically trivial.

Proof. Suppose to the contrary that $[g] \in \operatorname{Ker} \iota_{A T}$. Since $\left.g\right|_{\partial R} \neq \mathrm{id}$, there is some $x_{0} \in \partial R$ such that $g\left(x_{0}\right) \neq x_{0}$. Choose an open interval $I \subset \partial R$ containing $x_{0}$ such that $g(I) \cap I=\emptyset$. By the correspondence under a universal covering map of $R$, we may identify $I$ with an interval $(-1,1)$ in $\mathbb{R}$ and $x_{0}$ with 0 . Consider a quasiconformal homeomorphism $f: R \rightarrow R$ such that $f$ is the identity on $\partial R-I$ and $f: I \rightarrow I$ is defined by $f(x)=\frac{3}{2} x+\frac{1}{2}$ on $(-1,0]$ and $f(x)=\frac{1}{2} x+\frac{1}{2}$ on $[0,1)$.

The quasisymmetry quotient

$$
m_{t}\left(f, x_{0}\right)=\frac{f(t)-f(0)}{f(0)-f(-t)}
$$

for $f$ defined at $x_{0} \in I$ satisfies $\lim _{t \rightarrow 0} m_{t}\left(f, x_{0}\right)=\frac{1}{3}$. On the other hand, since $[g]$ is assumed to be asymptotically trivial, both $g$ and $f g f^{-1}$ are homotopic to asymptotically conformal automorphisms of $R$ relative to $\partial R$. Then, by [7] (see also [14]), the quasisymmetry quotients for $g$ and $f g f^{-1}$ tend to 1 everywhere on $\partial R$ as $t \rightarrow 0$. In particular,

$$
\lambda=\lim _{t \rightarrow 0} m_{t}\left(f g f^{-1}, f g^{-1}\left(x_{0}\right)\right)=1 .
$$

Since $g^{-1}\left(x_{0}\right) \notin I$ and $f$ is the identity outside $I$, we see that $\lambda$ is actually equal to $\lim _{t \rightarrow 0} m_{t}\left(f g, g^{-1}\left(x_{0}\right)\right)$. However, by using $\lim _{t \rightarrow 0} m_{t}\left(g, g^{-1}\left(x_{0}\right)\right)=1$ as well as the piecewise linearity of $f$, we obtain a contradicting value $\lambda=\frac{1}{3}$. This completes the proof.

\section{Proof of the FiXed POINT TheOREM}

In this section, we prove the fixed point theorem on the asymptotic Teichmüller space (Theorem 2.7). A rough idea of the proof is as follows. For each element of a given finite subgroup $\hat{\Gamma} \subset \operatorname{Mod}_{A T}(R)$, we consider the corresponding mapping class of $R$. Since there are finitely many, we can choose a sufficiently large topologically finite subsurface $\widetilde{V}$ of $R$ that is homotopically invariant under the subgroup generated by these mapping classes. (Actually, we need more arguments for this claim.) We want to see that its action restricted to $R-\widetilde{V}$ is reduced to a finite group isomorphic to $\hat{\Gamma}$. Since the ambiguity of choosing the mapping classes from $\hat{\Gamma}$ consists only of asymptotically trivial ones, Theorem 3.2 says that they are trivial outside some topologically finite subsurface. Thus we have a finite mapping class group $G$ acting on $R-\widetilde{V}$. Then by considering a certain kind of Teichmüller space for $R-\widetilde{V}$, we can find a common fixed point of $G$ on this space. By ignoring the action on a compact part in $\widetilde{V}$, we see that $\hat{\Gamma}$ has a fixed point in $A T(R)$. 
Proof of Theorem 2.7. We take an arbitrary finite subgroup $\hat{\Gamma}$ of $\operatorname{Mod}_{A T}(R)$ and number all its elements by $\left\{\hat{\gamma}_{i}\right\}_{i=1}^{n}$, where $\hat{\gamma}_{1}=[\mathrm{id}]_{* *}$. For each $i$, we choose a quasiconformal mapping class $\left[g_{i}\right] \in \operatorname{MCG}(R)\left(\left[g_{1}\right]=[\mathrm{id}]\right)$ such that $\iota_{A T}\left(\left[g_{i}\right]\right)=\hat{\gamma}_{i}$ and fix it. For any $i$ and $j$, there exists a unique $k=k(i, j)$ such that $\hat{\gamma}_{i} \hat{\gamma}_{j}=\hat{\gamma}_{k}$. Then an asymptotically trivial mapping class $\left[h_{i j}\right] \in \operatorname{Ker} \iota_{A T}$ is so determined that $\left[g_{i}\right]\left[g_{j}\right]=\left[h_{i j}\right]\left[g_{k}\right]$.

Since $R$ satisfies the weak bounded geometry condition, we have $\operatorname{Ker} \iota_{A T}=$ $G_{\infty}(R)$ by Theorem 3.2 . Then $\left[h_{i j}\right]$ for any $i$ and $j$ is an essentially trivial mapping class. Thus there exists a topologically finite subsurface $V_{i j}$ of finite area in $R$ such that the restriction $\left.h_{i j}\right|_{R-V_{i j}}: R-V_{i j} \rightarrow R$ is homotopic to the inclusion $\left.\operatorname{map} \mathrm{id}\right|_{R-V_{i j}}: R-V_{i j} \hookrightarrow R$ relative to the ideal boundary at infinity. We take a topologically finite subsurface $V_{0}$ of finite area so that all $V_{i j}(1 \leq i, j \leq n)$ are contained in $V_{0}$. Then, the restriction $\left.h_{i j}\right|_{R-V_{0}}: R-V_{0} \rightarrow R$ is homotopic to the inclusion map id $\left.\right|_{R-V_{0}}: R-V_{0} \hookrightarrow R$ relative to the ideal boundary at infinity for any $i$ and $j$.

Take a representative $g_{i}$ of each $\left[g_{i}\right]\left(g_{1}=\mathrm{id}\right)$ and choose a topologically finite subsurface $V$ of finite area that contains all $g_{i} g_{j}\left(V_{0}\right)(1 \leq i, j \leq n)$. In particular, $V$ satisfies $g_{i}(V) \cap V \neq \emptyset$ for every representative $g_{i}$.

Take the union

$$
\widetilde{V}=g_{1}(V)_{*} \cup \cdots \cup g_{n}(V)_{*}
$$

and consider the topologically finite subsurface $\widetilde{V}_{*}$ with geodesic boundary. We see that this is homotopically $g_{i}$-invariant for each $i$; in other words, $g_{i}(\widetilde{V})_{*}=\widetilde{V}_{*}$. This can be verified as follows. Since the restriction of $h_{i j}$ to each connected component of $R-V_{0}$ is homotopic to the inclusion map for any $i$ and $j$, and since both $g_{i} g_{j}(V)$ and $g_{k}(V)$ for $k=k(i, j)$ contain $V_{0}$, we have $g_{i} g_{j}(V)_{*}=g_{k}(V)_{*}$. Hence $\left\{g_{i} g_{j}(V)_{*}\right\}_{j=1}^{n}=\left\{g_{k}(V)_{*}\right\}_{k=1}^{n}$ for each $i$. Note that $\widetilde{V}_{*}$ can be characterized as the minimal subsurface with geodesic boundary that contains all $g_{1}(V)_{*}, \ldots, g_{n}(V)_{*}$. Then this is ready to show $g_{i}(\widetilde{V})_{*}=\widetilde{V}_{*}$. By replacing the representative $g_{i}$ of $\left[g_{i}\right]$, we may assume that $g_{i}\left(\widetilde{V}_{*}\right)=\widetilde{V}_{*}$.

Let $\left\{W_{*}^{(l)}\right\}_{l=1}^{m}$ be the family of all connected components of $R-\widetilde{V}_{*}$. We form the double $\widehat{W}^{(l)}$ of each $W_{*}^{(l)}$ with respect to the geodesic boundary $\partial W_{*}^{(l)}$. Extend $\left.g_{i}\right|_{R-\widetilde{V}_{*}}$ to a quasiconformal automorphism $\hat{g}_{i}$ of the union $S=\bigcup_{l=1}^{m} \widehat{W}^{(l)}$ of the Riemann surfaces by reflection. Then $\hat{g}_{i} \hat{g}_{j}$ is homotopic to $\hat{g}_{k}$ for $k=k(i, j)$ on each component $\widehat{W}^{(l)}$ of $S$ relative to the ideal boundary at infinity. We consider the mapping classes $\left[\hat{g}_{i}\right]$ on $S$ and define a map $\varepsilon: \hat{\Gamma} \rightarrow \operatorname{MCG}(S)$ by the correspondence $\hat{\gamma}_{i} \mapsto\left[\hat{g}_{i}\right]$. Since $\varepsilon$ is an injective homomorphism by the definition of $k(i, j)$, we see that the image $G=\left\{\left[\hat{g}_{i}\right]\right\}_{i=1}^{n}$ constitutes a finite subgroup of $\operatorname{MCG}(S)$.

The quasiconformal mapping class group $\operatorname{MCG}(S)$ acts on the product Teichmüller space $T(S)=\prod_{l=1}^{m} T\left(\widehat{W}^{(l)}\right)$ in the same manner as usual. Then $G$ has a common fixed point $\left(p_{1}, \ldots, p_{m}\right)$ in $T(S)$. Indeed, for some $l=1, \ldots, m$, we consider the stabilizer subgroup $G_{l}$ of $G$ preserving $\widehat{W}^{(l)}$ and utilize Theorem 2.3 to obtain a fixed point $p_{l}$ of $G_{l}$ in $T\left(\widehat{W}^{(l)}\right)$. Then we pick up the images of $p_{l}$ as the fixed points for the conjugates of $G_{l}$ in $G$, which are the stabilizer subgroups for the images of $\widehat{W}^{(l)}$. We apply this process to all possible components to assign the fixed point to $T\left(\widehat{W}^{(l)}\right)$ for every $l$. Giving each $\widehat{W}^{(l)}$ the complex structure corresponding 
to $p_{l}$, we can realize the mapping class group $G$ as a conformal automorphism group of the corresponding Riemann surfaces $S_{\left(p_{1}, \ldots, p_{m}\right)}$.

By restricting the complex structure $p_{l}$ to $W_{*}^{(l)}$ for each $l$, we have a point $\hat{p}$ of the asymptotic Teichmüller space $A T(R)$. Since the mapping class $\left[g_{i}\right]$ has a representative that is conformal outside $\widetilde{V}_{*}$ with respect to this complex structure, we see that each $\hat{\gamma}_{i}=\iota_{A T}\left(\left[g_{i}\right]\right)$ fixes the point $\hat{p}$.

In light of Theorem 2.3. we explore a problem asking whether we can extend Theorem 2.7 to the statement that there exists a common fixed point in $A T(R)$ if the orbit of a subgroup of $\operatorname{Mod}_{A T}(R)$ is bounded.

In [23], we constructed a Riemann surface $R$ not satisfying the upper bound condition so that $\operatorname{Mod}_{A T}(R)$ has a common fixed point in $A T(R)$. (Moreover, in [24], we gave an example where $\operatorname{Mod}_{A T}(R)$ acts on $A T(R)$ trivially.) On the other hand, it was proved in [10, Theorem 3.3] that $\operatorname{Mod}_{A T}(R)$ does not have a common fixed point in $A T(R)$ if $R$ satisfies the upper bound condition. This means that the asymptotic version of the fixed point theorem is not always true for the entire asymptotic Teichmüller modular group.

\section{REALIZATION IN THE PERIPHERAL QUASICONFORMAL AUTOMORPHISM GROUP}

Theorem 2.7 induces two asymptotic Nielsen realization theorems. In this section, we give one of them, which is for the asymptotic Teichmüller modular group. For its statement, we introduce the following concept.

Definition 5.1. We say that two quasiconformal automorphisms of a Riemann surface $R$ are peripherally equivalent if they coincide outside some topologically finite subsurface of finite area in $R$. The peripheral quasiconformal automorphism group $\mathrm{QC}_{e}(R)$ is the group of all peripheral equivalence classes $(g)_{e}$ of quasiconformal automorphisms $g$ of $R$. Furthermore, the peripheral conformal automorphism group $\operatorname{Conf}_{e}(R)$ is the subgroup of $\mathrm{QC}_{e}(R)$ consisting of all peripheral equivalence classes that have representatives conformal outside some topologically finite subsurface of finite area in $R$.

Let $e: \mathrm{QC}(R) \rightarrow \mathrm{QC}_{e}(R)$ be the homomorphism given by the projection $g \mapsto$ $(g)_{e}$. It is clear that $e$ splits the surjective homomorphism $\iota_{A T} \circ q: \mathrm{QC}(R) \rightarrow$ $\operatorname{Mod}_{A T}(R)$ into a surjective homomorphism $q_{e}: \mathrm{QC}_{e}(R) \rightarrow \operatorname{Mod}_{A T}(R)$. Hence we have the following commutative diagram:

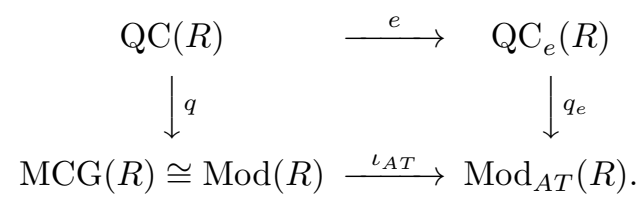

The Nielsen realization theorem for the asymptotic Teichmüller modular group can be stated as follows.

Theorem 5.2. Let $R$ be a Riemann surface satisfying the weak bounded geometry condition. Then every finite subgroup $\hat{\Gamma}$ of $\operatorname{Mod}_{A T}(R)$ can be realized as the peripheral conformal automorphism group $\operatorname{Conf}_{e}\left(R_{p}\right)$ of some Riemann surface $R_{p}$ quasiconformally equivalent to $R$. In particular, there exists a homomorphism $\mathcal{E}_{e}: \hat{\Gamma} \rightarrow \mathrm{QC}_{e}(R)$ such that $q_{e} \circ \mathcal{E}_{e}=\left.\mathrm{id}\right|_{\hat{\Gamma}}$. 
Proof. By Theorem 2.7 every finite subgroup $\hat{\Gamma}$ of $\operatorname{Mod}_{A T}(R)$ has a common fixed point $\hat{p}$ in $A T(R)$. Let $R_{p}$ be the Riemann surface corresponding to $p \in T(R)$ with $\alpha(p)=\hat{p}$. Then, for each element $\hat{\gamma} \in \hat{\Gamma}$, we have an asymptotically conformal automorphism $g$ of $R_{p}$ as a representative in the mapping class $[g] \in \mathrm{MCG}(R)$ satisfying $\iota_{A T}([g])=\hat{\gamma}$. Moreover, by the proof of Theorem 2.7 these asymptotically conformal automorphisms $g$ are actually conformal outside some topologically finite

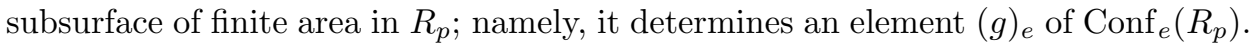

We note that such an element $(g)_{e} \in \operatorname{Conf}_{e}\left(R_{p}\right)$ is uniquely determined for each $\hat{\gamma} \in \hat{\Gamma}$. Indeed, suppose that there are two such elements $\left(g_{1}\right)_{e}$ and $\left(g_{2}\right)_{e}$ in $\operatorname{Conf}_{e}\left(R_{p}\right)$. The difference of their mapping classes is an asymptotically trivial one in Ker $\iota_{A T}$. Then, by Theorem 3.2 there is some topologically finite subsurface $V$ of finite area in $R_{p}$ such that the restriction of $g_{1} \circ g_{2}^{-1}$ to each connected component $U$ of the complement $R_{p}-V$ is conformal and homotopic to the inclusion map $\left.\mathrm{id}\right|_{U}: U \hookrightarrow R$ relative to the ideal boundary at infinity. We may assume that $U$ is topologically infinite. By [17, we see that $g_{1} \circ g_{2}^{-1}$ is precisely the identity on $U$. This shows that $\left(g_{1}\right)_{e}=\left(g_{2}\right)_{e}$.

Since the groups $\mathrm{QC}_{e}(R)$ and $\mathrm{QC}_{e}\left(R_{p}\right)$ are quasiconformally conjugate, the correspondence $\hat{\gamma} \mapsto(g)_{e}$ induces the homomorphism $\mathcal{E}_{e}: \hat{\Gamma} \rightarrow \mathrm{QC}_{e}(R)$ such that $q_{e} \circ \mathcal{E}_{e}=\left.\mathrm{id}\right|_{\hat{\Gamma}}$.

\section{REALiZATION IN THE AUTOMORPHisM GROUP OF THE ROYDEN BOUNDARY}

In this section, we give another asymptotic Nielsen realization theorem for the boundary geometric automorphism group of the asymptotic Teichmüller space. The asymptotic Teichmüller modular group is determined by the action of the group of quasiconformal automorphisms near the infinity of the Riemann surface. We extend this action to a certain ideal boundary of the Riemann surface and investigate the realization problem with respect to a homomorphism from the group of such boundary automorphisms to the biholomorphic automorphism group of the asymptotic Teichmüller space.

For a Riemann surface $R$, the Royden algebra $M(R)$ is a complex Banach algebra consisting of all bounded continuous functions $f$ on $R$ that are differentiable in the distribution sense with the Dirichlet integral $D(f)=\int_{R} d f \wedge * \overline{d f}$ finite. The norm is defined by $\|f\|=\sup |f|+D(f)^{1 / 2}$. There exists a compact Hausdorff space $R^{*}$ containing $R$ as an open and dense subset such that each function $f \in M(R)$ can be extended to a continuous function on $R^{*}$ and every pair of points in $R^{*}$ is separable by a function in $M(R)$. Then $R^{*}$ is uniquely determined up to homeomorphisms that are the identity on $R$. This is called the Royden compactification of $R$ and the boundary $d R=R^{*}-R$ is called the Royden boundary of $R$. An important property of the Royden compactification is that every quasiconformal homeomorphism $f$ of $R$ onto another Riemann surface $R^{\prime}$ extends continuously to a homeomorphism $\bar{f}: d R \rightarrow d R^{\prime}$. See [28, Chapter III] for details.

We deal with a smaller subset $d_{0} R$ of the Royden boundary $d R$ as in [29], which is obtained by removing all boundary points corresponding to punctures of $R$. More precisely, $d_{0} R$ is defined as follows. There is a canonical continuous map $\pi$ from the Royden boundary $d R$ onto the space of all topological ends of $R$, which extends continuously to the identity of $R$. The inverse image $\pi^{-1}(a)$ of a topological end $a$ corresponding to a puncture constitutes a connected component of $d R$. We remove 
all such connected components $\pi^{-1}(a)$ from $d R$ and define the remaining compact subset as $d_{0} R$, which we call the non-cuspidal Royden boundary. Note that a neighborhood of $d R$ in $R$ is given by the complement of a compact subsurface $V$ of $R$, whereas a neighborhood of $d_{0} R$ in $R$ is given by the union of the non-cuspidal connected components of $R-V$, which is the complement of some topologically finite subsurface of finite area in $R$. Since a quasiconformal homeomorphism $f$ : $R \rightarrow R^{\prime}$ preserves punctures, its extension $\bar{f}$ preserves the non-cuspidal Royden boundary, that is, $\bar{f}\left(d_{0} R\right)=d_{0} R^{\prime}$.

The converse property for the above extendability of quasiconformal homeomorphisms to the Royden boundary is also true in a certain sense, and we know that, if a homeomorphism $\bar{f}: d R \rightarrow d R^{\prime}$ is the restriction of a homeomorphism of $R^{*}$ onto $R^{\prime *}$ that is orientation-preserving on $R$, then $\bar{f}$ extends continuously to a quasiconformal homeomorphism $f: R \rightarrow R^{\prime}$. This was proved in [27]. In fact, local extendability is essential for its proof, and we can formulate this property for homeomorphic automorphisms of the non-cuspidal Royden boundary $d_{0} R$ as in [29, Proposition 3].

Proposition 6.1. Let $\bar{g}$ be a homeomorphic automorphism of $d_{0} R$ extending continuously to an orientation-preserving homeomorphism $g$ of some neighborhood of $d_{0} R$ in $R$ into $R$. Then there exists a topologically finite subsurface $V$ of finite area in $R$ such that $g$ is quasiconformal on the complement $R-V$.

The homeomorphism $g$ as in Proposition 6.1 is called a supporting map for $\bar{g}$. Note that the image of the neighborhood of $d_{0} R$ by $g$ is also a neighborhood of $d_{0} R$ in $R$. We say that a homeomorphic automorphism $\bar{g}$ of $d_{0} R$ is extendable if there is a supporting map for $\bar{g}$. We denote by $\operatorname{Homeo}^{*}\left(d_{0} R\right)$ the group of all extendable homeomorphic automorphisms of $d_{0} R$. Proposition 6.1 says that, for every element $\bar{g}$ of Homeo* $\left(d_{0} R\right)$, its supporting map $g$ is always quasiconformal on $R-V$ for some topologically finite subsurface $V$ of finite area in $R$. Since $g(R-V)$ is a neighborhood of $d_{0} R$ in $R$, we see that $R-g(R-V)$ is also topologically finite.

Let $\nu: \mathrm{QC}_{e}(R) \rightarrow$ Homeo*$^{*}\left(d_{0} R\right)$ be a homomorphism defined by extending a quasiconformal automorphism of $R$ to $d_{0} R$. Since an element of $\mathrm{QC}_{e}(R)$ has ambiguity on topologically finite subsurfaces of finite area, it cannot determine an automorphism of the entire boundary $d R$ uniquely, but the restriction to $d_{0} R$ kills such ambiguity to define the unique element of $\operatorname{Homeo}^{*}\left(d_{0} R\right)$.

The homomorphism $\nu$ is injective but not surjective. The reason why $\nu$ is not surjective is that a supporting map does not necessarily extend to the entire surface $R$. An example has been given in [29, Theorem 4]. Alternatively, we may consider a situation where $R$ has compact subsurfaces $V_{*}$ and $V_{*}^{\prime}$ with geodesic boundary such that $V_{*} \subsetneq V_{*}^{\prime}$ and there is a quasiconformal homeomorphism $g: R-V_{*} \rightarrow R-V_{*}^{\prime}$. Then $g$ extends to $\bar{g} \in$ Homeo $^{*}\left(d_{0} R\right)$ but not to a quasiconformal automorphism of $R$. In contrast, the injectivity of $\nu$ can be shown by a usual argument as follows.

Proposition 6.2. Let $g$ be a supporting map for $\bar{g} \in$ Homeo* $^{*}\left(d_{0} R\right)$. If $\bar{g}$ is the identity, then there exists a topologically finite subsurface $V$ of finite area in $R$ such that $g$ is the identity on $R-V$.

Proof. Assume that $g$ is not the identity outside any topologically finite subsurface $V$ of finite area in $R$. Then we can choose a discrete sequence of points $\left\{z_{n}\right\}_{n=1}^{\infty}$ in $R$ tending to the infinity of $R$, but not to any puncture such that $Z=\left\{z_{n}\right\}$ and $g(Z)=\left\{g\left(z_{n}\right)\right\}$ are disjoint. Then it is easy to construct a function $\varphi$ in the 
Royden algebra $M(R)$ such that $\varphi\left(z_{n}\right)=1$ and $\varphi\left(g\left(z_{n}\right)\right)=0$ for every $n$. Indeed, choose a disk neighborhood $U_{n} \subset R-V$ of $z_{n}$ such that $U_{n} \cap(Z \cup g(Z))$ consists only of $z_{n}$ for each $n$. Take a Riemann map $\zeta_{n}$ of $U_{n}$ onto the unit disk $\Delta$ such that $\zeta_{n}\left(z_{n}\right)=0$. We consider a function

$$
h_{n}(\zeta)=\min \left\{-\frac{\log |\zeta|}{n^{2}}, 1\right\} \geq 0 \quad\left(h_{n}(0)=1\right)
$$

on $\Delta$. An easy calculation shows that the Dirichlet integral of $h_{n}$ is $D\left(h_{n}\right)=2 \pi / n^{2}$. Define the function $\varphi(z)$ on $R$ so that $\varphi(z)=h_{n}\left(\zeta_{n}(z)\right)$ if $z \in U_{n}$ for some $n$ and otherwise $\varphi(z)=0$. Then $\varphi$ is continuous and bounded with the Dirichlet integral $D(\varphi)=\pi^{3} / 3$, and hence belongs to $M(R)$. By construction, we see that $\varphi\left(z_{n}\right)=1$ and $\varphi\left(g\left(z_{n}\right)\right)=0$.

Since every infinite set in a compact Hausdorff space has an accumulation point, $Z$ has an accumulation point $z_{\infty}$ in $d_{0} R$. Then $\bar{g}\left(z_{\infty}\right)$ is an accumulation point of $g(Z)$. If the extension $\bar{g}$ of $g$ is the identity on $d_{0} R$, then $\bar{g}\left(z_{\infty}\right)=z_{\infty}$. This implies that $Z$ and $g(Z)$ have the common accumulation point $z_{\infty}$. On the other hand, since $\varphi \in M(R)$ extends to a continuous function on $R^{*}$, the sets $X_{1}=\{z \in$ $\left.R^{*} \mid \varphi(z)=1\right\}$ and $X_{0}=\left\{z \in R^{*} \mid \varphi(z)=0\right\}$ are both closed. Since $Z \subset X_{1}$ and $g(Z) \subset X_{0}$, the common accumulation point $z_{\infty}$ belongs to both $X_{1}$ and $X_{0}$. This contradiction proves the statement.

Next, we consider a representation of $\operatorname{Homeo}^{*}\left(d_{0} R\right)$ in the biholomorphic automorphism group $\operatorname{Aut}(A T(R))$ of the asymptotic Teichmüller space $A T(R)$. This has also been introduced in [29]. A quasiconformal supporting map $g: R-V \rightarrow R$ for $\bar{g} \in$ Homeo $^{*}\left(d_{0} R\right)$ induces a biholomorphic automorphism of $A T(R)$ by $[[f]] \mapsto$ $\left[\left[f \circ g^{-1}\right]\right]$ for each element $[[f]]$ in $A T(R)$. Although the quasiconformal homeomorphism $f \circ g^{-1}$ is defined only on $g(R-V)$, it determines the asymptotic equivalence class $\left[\left[f \circ g^{-1}\right]\right]$ because the value of $f \circ g^{-1}$ on the topologically finite subsurface $R-g(R-V)$ of finite area is negligible and hence it can be arbitrarily given. In this manner, we have a homomorphism $q_{b}$ : $\operatorname{Homeo}^{*}\left(d_{0} R\right) \rightarrow \operatorname{Aut}(A T(R))$.

Definition 6.3. The boundary geometric automorphism group is the subgroup of $\operatorname{Aut}(A T(R))$ consisting of all elements induced by supporting maps for $\operatorname{Homeo}^{*}\left(d_{0} R\right)$ and is denoted by $\operatorname{Aut}^{*}(A T(R))$ :

$$
\operatorname{Aut}^{*}(A T(R))=q_{b}\left(\operatorname{Homeo}^{*}\left(d_{0} R\right)\right) .
$$

It is clear that the asymptotic Teichmüller modular group $\operatorname{Mod}_{A T}(R)$ is contained in $\operatorname{Aut}^{*}(A T(R))$; namely, there is an inclusion map $i: \operatorname{Mod}_{A T}(R) \rightarrow$ $\operatorname{Aut}^{*}(A T(R))$.

Remark 6.4. We conjecture that the boundary geometric automorphism group Aut $^{*}(A T(R))$ actually coincides with the entire biholomorphic automorphism group $\operatorname{Aut}(A T(R))$. This claim corresponds to the fact for Teichmüller spaces that the Teichmüller modular group $\operatorname{Mod}(R)$ coincides with $\operatorname{Aut}(T(R))$. We also expect $\operatorname{Mod}_{A T}(R)$ to be a proper subgroup of $\operatorname{Aut}^{*}(A T(R))$. Actually, it is proved in [11] that $\operatorname{Mod}_{A T}(R)$ is geometrically isomorphic to the biholomorphic automorphism group $\operatorname{Aut}(I T(R))$ of the intermediate Teichmüller space $I T(R)$ if $R$ satisfies the bounded geometry condition. 
Now, given the following commutative diagram,

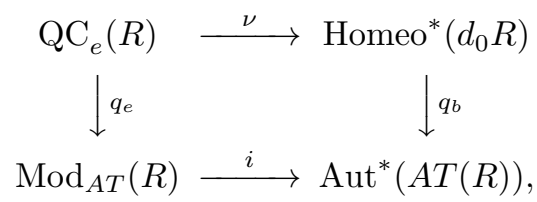

we formulate the Nielsen realization theorem for the surjective homomorphism $q_{b}$ : Homeo* $^{*}\left(d_{0} R\right) \rightarrow$ Aut $^{*}(A T(R))$ and give a proof for it.

Theorem 6.5. Let $R$ be a Riemann surface satisfying the weak bounded geometry condition. Then, for every finite subgroup $\widetilde{\Gamma}$ of $\operatorname{Aut}^{*}(A T(R))$, there exists a homomorphism $\mathcal{E}_{b}: \widetilde{\Gamma} \rightarrow \operatorname{Homeo}^{*}\left(d_{0} R\right)$ such that $q_{b} \circ \mathcal{E}_{b}=\left.\mathrm{id}\right|_{\widetilde{\Gamma}}$.

As is shown below, the essential part of this theorem lies in the following lemma. Then Theorem 6.5 will be just an interpretation of Theorem 5.2 , the realization for $q_{e}: \mathrm{QC}_{e}(R) \rightarrow \operatorname{Mod}_{A T}(R)$.

Lemma 6.6. Let $R$ be a Riemann surface satisfying the weak bounded geometry condition. Then every element $\tilde{\gamma}$ of $\operatorname{Aut}^{*}(A T(R))$ of finite order belongs to $\operatorname{Mod}_{A T}(R)$.

Proof. We take a quasiconformal supporting map $g$ inducing $\tilde{\gamma}$, which maps $R-V_{0}$ into $R$ for some topologically finite subsurface $V_{0}$ of finite area in $R$. If $\tilde{\gamma}$ is of order $n$, then $g^{n}$ induces the trivial action on $A T(R)$. By the same reason as the assertion that an asymptotically trivial mapping class is essentially trivial (Theorem 3.2), we see that there exists a topologically finite subsurface $V$ of finite area containing $V_{0}$ such that $\left.g^{n}\right|_{R-V}$ is homotopic to the inclusion map id| $\left.\right|_{R-V}: R-V \hookrightarrow R$ relative to the ideal boundary at infinity.

Similarly to the proof of Theorem 2.7 we consider $\bigcap_{i=0}^{n-1} g^{i}(R-V)_{*}$, whose complement in $R$ is defined to be $\tilde{V}$. Since $g^{n}(R-V)_{*}=(R-V)_{*}$, we see that $g(R-\widetilde{V})_{*}=(R-\widetilde{V})_{*}$. By replacing $g$, we may assume that $g$ preserves $(R-\widetilde{V})_{*}$. Then we can extend $g$ to the topologically finite subsurface $\widetilde{V}_{*}$ with geodesic boundary to construct a quasiconformal automorphism $\tilde{g}$ of $R$. Hence $\tilde{\gamma}$ is induced by $\tilde{g}$, which means that $\tilde{\gamma} \in \operatorname{Mod}_{A T}(R)$.

We are ready to prove our theorem.

Proof of Theorem 6.5. Let $\widetilde{\Gamma}$ be a finite subgroup of $\operatorname{Aut}^{*}(A T(R))$. By Lemma 6.6. each element of $\widetilde{\Gamma}$ actually belongs to $\operatorname{Mod}_{A T}(R)$, and hence $\widetilde{\Gamma} \subset \operatorname{Mod}_{A T}(R)$. We define a homomorphism $\mathcal{E}_{b}$ of $\widetilde{\Gamma}$ to be the composition of $\mathcal{E}_{e}: \widetilde{\Gamma} \rightarrow \mathrm{QC}_{e}(R)$ obtained in Theorem 5.2 and $\nu: \mathrm{QC}_{e}(R) \rightarrow \operatorname{Homeo}^{*}\left(d_{0} R\right)$. From $q_{e} \circ \mathcal{E}_{e}=\left.\mathrm{id}\right|_{\widetilde{\Gamma}}$, it follows that

$$
q_{b} \circ \mathcal{E}_{b}=q_{b} \circ \nu \circ \mathcal{E}_{e}=q_{e} \circ \mathcal{E}_{e}=\left.\mathrm{id}\right|_{\widetilde{\Gamma}} .
$$

This proves the statement.

\section{The AUtomorphism GROUP OF THE SPACE OF THE ENDS}

We conclude this paper by commenting that the sequence of homomorphisms

$$
\mathrm{QC}(R) \rightarrow \mathrm{QC}_{e}(R) \rightarrow \text { Homeo }^{*}\left(d_{0} R\right)
$$


for raw mappings and the sequence of homomorphisms

$$
\operatorname{Mod}(R) \rightarrow \operatorname{Mod}_{A T}(R) \rightarrow \operatorname{Aut}^{*}(A T(R))
$$

for mapping classes meet into the group of homeomorphic automorphisms of the space of the ends of $R$. We use a slightly finer concept of ends as follows, which has been introduced in 12 .

The end compactification $\bar{R}^{\delta}$ of a Riemann surface $R$ is given by adding all the ends of $R$ and by providing the canonical topology. Here an end means a topological end if $R$ has no ideal boundary at infinity. However, if $R$ has the ideal boundary at infinity $\partial R$, we first consider the double $\hat{R}$ of $R$ with respect to $\partial R$ and then take the closure of $R$ in the end compactification of $\hat{R}$, which we define to be $\bar{R}^{\delta}$. The boundary $\bar{R}^{\delta}-R$ is denoted by $\delta R$. As before, we remove all ends corresponding to punctures from $\delta R$ and denote the space of all non-cuspidal ends by $\delta_{0} R$. A supporting map $g$ for $\bar{g} \in \operatorname{Homeo}^{*}\left(d_{0} R\right)$ extends to a homeomorphic automorphism of $\delta_{0} R$, which defines a homomorphism $\kappa$ : Homeo* $\left(d_{0} R\right) \rightarrow \operatorname{Homeo}\left(\delta_{0} R\right)$. Note that there is a continuous surjective map $\hat{\pi}: d R \rightarrow \delta R$ from the Royden boundary to the space of the ends that extends to the identity of $R$. The restriction of $\hat{\pi}$ to $d_{0} R$ is compatible with the above homomorphism $\kappa$.

On the other hand, since each mapping class $[g] \in \operatorname{MCG}(R)$ induces a homeomorphic automorphism of $\delta_{0} R$, there is a well-defined homomorphism $\iota_{\delta_{0}} R: \operatorname{MCG}(R) \rightarrow$ Homeo $\left(\delta_{0} R\right)$. The kernel of $\iota_{\delta_{0}}$ is defined to the pure mapping class group. In [9], it has been shown that $\operatorname{Ker} \iota_{A T} \subset \operatorname{Ker} \iota_{\delta_{0} R}$, that is, the asymptotically trivial mapping class group is contained in the pure mapping class group, though the definition of $\delta_{0} R$ used for this group is slightly coarser than the one above. The inclusion $\operatorname{Ker} \iota_{A T} \subset \operatorname{Ker} \iota_{\delta_{0} R}$ would yield a homomorphism of $\operatorname{Mod}_{A T}(R)$ to Homeo $\left(\delta_{0} R\right)$.

In what follows, we will show the existence of a homomorphism from the larger group Aut $^{*}(A T(R))$ to $\operatorname{Homeo}\left(\delta_{0} R\right)$. For this purpose, we prove the following inclusion relation under the new definition of the end compactification.

Lemma 7.1. Ker $q_{b} \subset \operatorname{Ker} \kappa$.

Proof. Let $\bar{g} \in$ Homeo $^{*}\left(d_{0} R\right)$ not belong to Ker $\kappa$. We take a supporting map $g$ for $\bar{g}$ defined on $R-V$, where $V$ is a topologically finite subsurface of finite area in $R$. Then there is an end $e \in \delta_{0} R$ such that $g(e) \neq e$. If $R$ has no ideal boundary at infinity, then there exists a neighborhood of $e$ whose intersection with $R$ is a topologically infinite subsurface $W$ in $R-V$ such that $g(W) \cap W=\emptyset$. In this case, $g$ induces a non-trivial automorphism of $A T(R)$ by the same argument as in 9 , Theorem 1.5] proving the inclusion $\operatorname{Ker} \iota_{A T} \subset \operatorname{Ker} \iota_{\delta_{0} R}$.

Next we assume that $R$ has ideal boundary at infinity $\partial R$. If $g$ extends to $\partial R$ as a non-identical map, then, by the same argument as Lemma 3.5 we see that $g$ induces a non-trivial automorphism of $A T(R)$. If $g$ is the identity on $\partial R$, then its extension to the boundary $\delta R$ of the end compactification is the identity on the closure $\overline{\partial R}^{\delta}$ of $\partial R$. Since $g(e) \neq e$ for some $e \in \delta_{0} R$, this means that $e$ is not in $\overline{\partial R}^{\delta}$. Then again we can find a topologically infinite subsurface $W$ of $R$ such that $g(W) \cap W=\emptyset$ and hence $g$ induces a non-trivial automorphism of $A T(R)$ by [9]. In all cases, we have shown that $\bar{g}$ does not belong to Ker $q_{b}$. 
Thus we have the following diagram by factorizing $\kappa$ into $q_{b}$ and $\kappa^{\prime}$ :

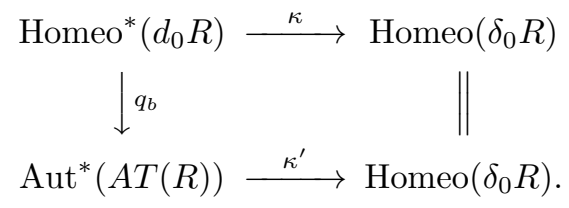

The existence of the homomorphism $\kappa^{\prime}: \operatorname{Aut}^{*}(A T(R)) \rightarrow \operatorname{Homeo}\left(\delta_{0} R\right)$ in particular verifies the following fact we have mentioned above.

Corollary 7.2. The pure mapping class group $\operatorname{Ker} \iota_{\delta_{0} R}$ contains the asymptotically trivial mapping class group $\operatorname{Ker} \iota_{A T}$.

This is the end of our supplementary comments.

\section{REFERENCES}

[1] C. J. Earle and F. P. Gardiner, Geometric isomorphisms between infinite dimensional Teichmüller spaces, Trans. Amer. Math. Soc. 348 (1996), 1163-1190. MR.1322950 (96h:32024)

[2] C. J. Earle, F. P. Gardiner and N. Lakic, Teichmüller spaces with asymptotic conformal equivalence, I.H.E.S., 1995, unpublished manuscript.

[3] C. J. Earle, F. P. Gardiner and N. Lakic, Asymptotic Teichmüller space, Part I: The complex structure, In the tradition of Ahlfors and Bers, Contemporary Math. 256 (2000), 17-38. MR:1759668(2001m:32029)

[4] C. J. Earle, F. P. Gardiner and N. Lakic, Asymptotic Teichmüller space, Part II: The metric structure, In the tradition of Ahlfors and Bers, III, Contemporary Math. 355 (2004), 187-219. MR2145063 (2006g:30078)

[5] C. J. Earle, V. Markovic and D. Šarić, Barycentric extension and the Bers embedding for asymptotic Teichmüller space, Complex manifolds and hyperbolic geometry, Contemporary Math. 311 (2002), 87-105. MR1940165 (2003i:30072)

[6] A. L. Epstein, Effectiveness of Teichmüller modular groups, In the tradition of Ahlfors and Bers, Contemporary Math. 256 (2000), 69-74. MR1759670 (2001a:30059)

[7] R. Fehlmann, Über extremale quasikonforme Abbildungen, Comment Math. Helv. 56 (1981), 558-580. MR656212 (83e:30024)

[8] E. Fujikawa, The order of periodic elements of Teichmüller modular groups, Tohoku Math. J. 57 (2005), 45-51. MR2113989 (2005j:30059)

[9] E. Fujikawa, Pure mapping class group acting on Teichmüller space, Conform. Geom. Dyn. 12 (2008), 227-239. MR.2466018 (2009j:30103)

[10] E. Fujikawa, Limit set of quasiconformal mapping class group on asymptotic Teichmüller space, Teichmüller theory and moduli problems, Lecture note series in the Ramanujan Math. Soc. 10 (2009), 167-178. MR2667555 (2011g:30097)

[11] E. Fujikawa and K. Matsuzaki, Stable quasiconformal mapping class groups and asymptotic Teichmüller spaces, Amer. J. Math. 133 (2011), 637-675. MR2808328

[12] E. Fujikawa, K. Matsuzaki and M. Taniguchi, Structure theorem for holomorphic selfcovers and its applications, Infinite dimensional Teichmüller space and moduli space, RIMS Kôkyûroku Bessatsu, Research Institute for Mathematical Sciences, Kyoto University, B17 (2010), 21-36. MR2560682 (2012b:30085)

[13] F. P. Gardiner and N. Lakic, Quasiconformal Teichmüller Theory, Mathematical Surveys and Monographs 76, Amer. Math. Soc., 2000. MR.1730906 (2001d:32016)

[14] F. P. Gardiner and D. P. Sullivan, Symmetric structure on a closed curve, Amer. J. Math. 114 (1992), 683-736. MR.1175689 (95h:30020)

[15] S. P. Kerckhoff, The Nielsen realization problem, Ann. of Math. (2) 117 (1983), 235-265. MR690845 (85e:32029)

[16] O. Lehto, Univalent Functions and Teichmüller Spaces, Graduate Texts in Math. 109, Springer, 1986. MR867407 (88f:30073)

[17] F. Maitani, On rigidity of an end under conformal mapping preserving the infinity homology basis, Complex Variables 24 (1994), 281-287. MR,1270318 (95e:30010) 
[18] V. Markovic, Biholomorphic maps between Teichmüller spaces, Duke Math. J. 120 (2003), 405-431. MR2019982 (2004h:30058)

[19] V. Markovic, Quasisymmetric groups, J. Amer. Math. Soc. 19 (2006), 673-715. MR.2220103 (2007c:37057)

[20] V. Markovic, Realization of the mapping class group by homeomorphisms, Invent. Math. 168 (2007), 523-566. MR2299561 (2008c:57033)

[21] V. Markovic and D. Sarić, The mapping class group cannot be realized by homeomorphisms, preprint.

[22] K. Matsuzaki, Inclusion relations between the Bers embeddings of Teichmüller spaces, Israel J. Math. 140 (2004), 113-124. MR2054840(2005e:30077)

[23] K. Matsuzaki, A countable Teichmüller modular group, Trans. Amer. Math. Soc. 357 (2005), 3119-3131. MR2135738(2006f:30052)

[24] K. Matsuzaki, A quasiconformal mapping class group acting trivially on the asymptotic Teichmüller space, Proc. Amer. Math. Soc. 135 (2007), 2573-2579. MR2302578 (2008d:30071)

[25] K. Matsuzaki, The action of elliptic modular transformations on asymptotic Teichmüller spaces, Proceedings of the international workshop on Teichmüller theory and moduli problems, Lecture Note Series in the Ramanujan Mathematical Society, 10 (2010), 481-488. MR2667568 (2011k:30054)

[26] S. Nag, The Complex Analytic Theory of Teichmüller Spaces, Canadian Math. Soc. Ser. of Monographs and Advanced Texts, A Wiley-Interscience Publication, John Wiley \& Sons, 1988. MR.927291 (89f:32040)

[27] M. Nakai, Existence of quasiconformal mappings between Riemann surfaces, Hokkaido Math. J. 10 (1981), Special Issue, 525-530. MR662318 (83f:30013)

[28] L. Sario and M. Nakai, Classification theory of Riemann surfaces, Die Grundlehren der mathematischen Wissenschaften 164, Springer, 1970. MR0264064 (41:8660)

[29] M. Taniguchi, The Teichmüller space of the ideal boundary, Hiroshima Math. J. 36 (2006), 39-48. MR 2213642(2007i:30065)

Department of Mathematics, Chiba University, Inage-Ku, Chiba 263-8522, Japan

E-mail address: fujikawa@math.s.chiba-u.ac.jp

Department of Mathematics, School of Education, Waseda University, Shinjuku, TOKYO 169-8050, JAPAN

E-mail address: matsuzak@waseda.jp 